\title{
The necessary and sufficient conditions for a probability distribution belongs to the domain of geometric attraction of standard Laplace distribution
}

\author{
Tran Loc Hung* , Phan Tri Kien
}

University of Finance and Marketing, Vietnam

\section{Correspondence}

Tran Loc Hung, University of Finance and Marketing, Vietnam

Email: thung@ufm.edu.vn

\section{History}

- Received: 2018-11-19

- Accepted: 2019-03-19

- Published: 2019-03-29

DOI :

https://doi.org/10.32508/stdj.v22i1.1049

\section{Check for updates}

\section{Copyright}

(.) VNU-HCM Press. This is an openaccess article distributed under the terms of the Creative Commons Attribution 4.0 International license.

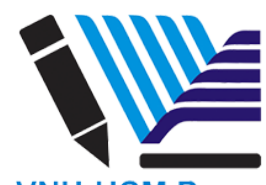

VNU-HCM Press

\begin{abstract}
The geometric sums have been arisen from the necessity to resolve practical problems in ruin probability, risk processes, queueing theory and reliability models, etc. Up to the present, the results related to geometric sums like asymptotic distributions and rates of convergence have been investigated by many mathematicians. However, in a lot of various situations, the results concerned domains of geometric attraction are still limitative. The main purpose of this article is to introduce concepts on the domain of geometric attraction of standard Laplace distribution. Using method of characteristic functions, the necessary and sufficient conditions for a probability distribution belongs to the domain of geometric attraction of standard Laplace distribution are shown. In special case, obtained result is a weak limit theorem for geometric sums of independent and identically distributed random variables which has been well-known as the second central limit theorem. Furthermore, based on the obtained results of this paper, the analogous results for the domains of geometric attraction of exponential distribution and Linnik distribution can be established. More generally, we may extend results to the domain of geometric attraction of geometrically strictly stable distributions.
\end{abstract}

Mathematics Subject Classification 2010: 60G50; 60F05; 60E07.

Key words: Geometric sums, Standard Laplace distribution, Domain of geometric attraction, Characteristic function, Geometrically infinitely divisible, Geometrically strictly stable.

\section{INTRODUCTION}

During the last several decades, the weak limit theorems for geometric sums have been become one of the most important problems in applied probability and related topics such as insurance risk theory, stochastic finance and queuing theory, etc. Klebanov et al. (1984) introduced the concepts on geometrically infinitely divisible (GID) distributions and geometrically strictly stable (GSS) distributions ${ }^{1}$. Up to now, the geometric random sums have been investigated by many mathematicians such as Kruglov and Korolev (1990), Kalashnikov (1997), Kotz et al. (2001), Kozubowski (2000), Kozubowski and Podrsky (2010),

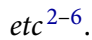

It is worth pointing out that, the class of geometrically strictly stable laws are closely related to the heavy tail distributions like exponential distribution, Laplace distribution and Linnik distribution ${ }^{7}$. Recently, some results on the weak limit theorems for geometric sums together with rates of convergence and its applications were published by Hung (2013), Teke and Deshmukh $(2014)^{8,9}$. However, in any situations, results related to the domain of geometric attractions are still restrictive. For a deeper discussion of this problem we refer the reader to Kruglov and Korolev $(1990)^{2}$ and Sandhya and Pillai (1999) ${ }^{10}$.

The main purpose of this paper is to show the necessary and sufficient conditions for the distribution function $F$ which belongs to the domain of geometric attraction of standard Laplace distribution by using method of characteristic functions. Furthermore, a weak limit theorem for geometric sums converging to the standard Laplace distribution is established.

The article is organized as follows. Some basic notations and auxiliary results will be presented in Preliminaries Section. The Main results Section devotes to present our main results (Theorem 3.1, Theorem 3.2 and Corollary 3.1). In Discussions Section, we have discussed how the main objective be solved by our method. Finally, some conclusions and acknowledgments will be stated in Conclusions and Acknowledgments Section. From now on, the notation $\underline{D}$ expresses converge in distribution and the set of real numbers is denoted by $\mathbb{R}=(-\infty,+\infty)$. Dev. J.; 22(1):143-146. 


\section{PRELIMINARIES}

Before stating the main theorems we first recall fundamental notions and some classical results that had been presented in references ${ }^{4,11,12}$. The characteristic function $f(t)$ of the random variable $X$ is defined in form

$$
f(t)=E\left(e^{i t X}\right), t \in \mathbb{R}
$$

With respect to the characteristic functions, we will recall following result which will useful for proofs of our main results $\left(\right.$ see $\left.^{1}\right)$.

Theorem 2.1 ( ${ }^{1}$, Proposition 8.44, p. 180). Let $E\left(|X|^{k}\right)<+\infty$. Then, the characteristic function of $X$ has the expansion

$f(u)=\sum_{j=1}^{k-1} \frac{(i u)^{j}}{j} E\left(X^{j}\right)+\frac{(i u)^{k}}{k !}\left[E\left(X^{k}\right)+\delta(u)\right]$,

where $\delta(u)$ denotes a function of $u$, such that for all $u$, $\lim _{u \rightarrow 0} \delta(u)=0$ and $|\delta(u)| \leq 3 E|X|^{k}$.

For $p \in(0,1)$, a random variable $v_{p}$ is said to be a geometric random variable with mean $1 / \mathrm{p}$, denoted by $v_{p} \sim G e o(p)$, if its probability distribution given as follows

$$
P\left(v_{p}=k\right)=p(1-p)^{k-1}, k=1,2, \ldots
$$

Let $\left\{X_{j}, j \geq 1\right\}$ be a sequence of independent and identically distributed (i.i.d.) random variables, independent of $v_{p}$. We write

$$
S_{v_{p}}=\sum_{j=1}^{v_{p}} X_{j}
$$

and it is called the geometric sums.

According to ${ }^{6}$, a random variable $Y$ is said to be a standard Laplace distributed random variable, denoted by $Y \sim \mathscr{L}(0,1)$, if its characteristic function is given as

$$
\varphi_{Y}(t)=\frac{1}{1+\frac{t^{2}}{2}}, t \in \mathbb{R}
$$

Note that, if $Y \sim \mathscr{L}(0,1)$ then $E(Y)=0$ and $E\left(Y^{2}\right)=$ 1. Moreover, the standard Laplace distribution is a special case of geometrically strictly stable distributions which was introduced by Klebanov et al. in 1984 $\left(\right.$ See $\left.^{4,6}\right)$.

\section{MAIN RESULTS}

Let $\left\{X_{j}, j \geq 1\right\}$ be a sequence of i.i.d. random variables with common distribution function $F(x)$ and corresponding characteristic function $f(t)$. We introduce the following notations.

Definition 3.1 A distribution function $F(x)$ is said to be geometrically attracted to standard Laplace distribution, if there exists the suitable positive constant $c(p)$, such that $c(p) \downarrow 0$ as $p \downarrow 0$ and

$$
c(p) \sum_{j=1}^{v_{p}} X_{j} \stackrel{D}{\rightarrow} Y \sim L(0,1) \text {, as } p \downarrow 0,
$$

where $v_{p}$ is a geometric random variable with mean $1 / p, p \in(0,1)$, independent of all $X_{j}$ for all $j \geq 1$.

Definition 3.2 The set of all distribution functions that are geometrically attracted to standard Laplace distribution is called the domain of geometric attraction of standard Laplace distribution and denoted by $D G A_{\mathscr{L}(0,1)}$.

The following theorem will show the necessary and sufficient conditions for the distribution function $F(x)$ which belongs to the domain of geometric attraction of standard Laplace distribution.

Theorem 3.1 Let $\left\{X_{j}, j \geq 1\right\}$ be a sequence of i.i.d. random variables with common distribution function $F(x)$ and corresponding characteristic function $f(t)$. The following statements are equivalent:

1. $F(x) \in D G A_{\mathscr{L}(0,1)}$;

2. The characteristic function $f(t)$ satisfies

$\lim _{p \rightarrow 0^{+}}\left\{\frac{1}{p}[1-f(c(p))]\right\}=\frac{1}{2} t^{2}, t \in \mathbb{R}$.

Proof. Since $v_{p} \sim G e o(p)$, let us denote by

$$
G_{v_{p}}(t)=\frac{p t}{1-(1-p) t}, t \in \mathbb{R}
$$

the generating function of geometric random variable $v_{p}$. Then, the characteristic function of the geometric random sum $S_{v_{p}}=\sum_{j=1}^{v_{p}} X j$ is defined by

$\varphi_{S_{v_{p}}}(t)=G_{v_{p}}[f(t)]=\frac{p f(t)}{1-(1-p) f(t)}, t \in \mathbb{R}$

Thus, the characteristic function of $c(p) S_{v_{p}}=$ $c(p) \sum_{j=1}^{v_{p}} X_{j}$ is defined as

$$
\varphi_{c(p) S_{v p}}(t)=\varphi_{S_{v p}}[c(p) t]=\frac{p f[c(p) t]}{1-(1-p) f[c(p) t]},
$$
$t \in \mathbb{R}$.

By the continuity of $f$ the property $f(0)=1$ and $c(p) \downarrow 0$ as $p \downarrow 0$ we have

$$
f[c(p) t] \rightarrow 1, \quad \text { as } p \downarrow 0 .
$$


Hence,

$$
\begin{aligned}
& \lim _{p \rightarrow 0^{+}} \varphi_{c(p) S_{v p}(t)=\lim _{p \rightarrow 0} \frac{p f[c(p) t]}{1-(1-p) f[c(p) t]}}=\lim _{p \rightarrow 0^{+}} \frac{\frac{1}{p}[1-(1-p) f(c(p))]}{1} \\
& =\lim _{p \rightarrow 0^{+}} \frac{1}{\frac{1}{p}[1-f(c(p))]+1} .
\end{aligned}
$$

Therefore, $F(x) \in D G A_{\mathscr{L}(0,1)}$ if and only if

$$
\lim _{p \rightarrow 0^{+}} \frac{1}{\frac{1}{p}[1-f(c(p))]+1}=\frac{1}{1+\frac{1}{2} t^{2}} .
$$

Equivalently,

$$
\lim _{p \rightarrow 0^{+}}\left\{\frac{1}{p}[1-f(c(p))]\right\}=\frac{1}{2} t^{2}, t \in \mathbb{R} .
$$

The proof is complete.

Additionally, if the sequence of i.i.d. random variables $X_{1}, X_{2}, \ldots$ has the moments $E\left(X_{1}\right)$ and $E\left(X_{1}^{2}\right)$ are finite, then its distribution function will belong to the domain of geometric attraction of standard Laplace distribution. This assertion will be evidenced by the following theorem.

Theorem 3.2. Let $X_{1}, X_{2}, \ldots$ be a sequence of i.i.d. random variables with the common distribution function $F(x), E\left(X_{1}\right)=0$ and $E\left(X_{1}^{2}\right)=1$. Assume that, the constant $c(p)$ satisfies the condition $\lim _{p \rightarrow 0^{+}} \frac{[c(p)]^{2}}{p}=1$.

Then, $F(x) \in D G A_{\mathscr{L}(0,1)}$.

Proof. Let $f(t)$ be the corresponding characteristic function of the distribution function $F(x)$. Using the hypothesis of this theorem and according to Theorem 2.1 , we can write

$$
\begin{array}{r}
f(w)=1+\frac{w i}{1 !} E\left(X_{1}\right)+\frac{(w i)^{2}}{2 !}\left[E\left(X_{1}^{2}\right)+R(w)\right] \\
=1-\frac{w^{2}}{2}[1+R(w)],
\end{array}
$$

where $R(w)$ denotes a bounded function of $w$ such that $R(w) \rightarrow 0$ as $w \rightarrow 0$.

Thus, for $w=c(p) t$, we obtain

$$
f(c(p) t)=1-\frac{\left.[c(p)]^{2} t^{2}\right)}{2}[1+R(c(p) t)],
$$

where $R(c(p)) \rightarrow 0$ as $p \downarrow 0$, for all $t \in \mathbb{R}$. Using the condition $\lim _{p \rightarrow 0^{+}} \frac{[c(p)]^{2}}{p}=1$, we have

$$
\begin{aligned}
& \lim _{p \rightarrow 0^{+}}\left\{\frac{1}{p}[1-f(c(p) t)]\right\} \\
& =\lim _{p \rightarrow 0^{+}} \frac{\left([c(p)]^{2} t^{2}\right)}{2 p}[1+R(c(p) t)] \\
& =\frac{1}{2} t^{2} .
\end{aligned}
$$

According to Theorem 3.1, it finishes the proof.

The following corollary could be considered as second central limit theorem.

Corollary 3.1 Let $X_{1}, X_{2}, \ldots$ be a sequence of i.i.d. random variables with the common distribution function $F(x) E\left(X_{1}\right)=0$ and $E\left(X_{1}^{2}\right)=1$. Then,

$$
p^{\frac{1}{2}} \sum_{j=1}^{v_{p}} X_{j} \stackrel{D}{\rightarrow} Y \sim \mathscr{L}(0,1) \text { as } p \downarrow 0 .
$$

Proof. Applying to Theorem 3.2 with $c(p)=p 1 / 2$ the proof is straight-forward.

\section{DISCUSSIONS}

There are various methods have been used in investigation of domains of attraction in probability theory like method of characteristic functions, method of linear operators or method of probability distances, etc. Especially, the method of characteristic functions is more effective. For this reason we have used the method of characteristic functions in this study and some results on the domain of geometric attraction of standard Laplace distribution in this research were obtained.

\section{CONCLUSIONS}

Based on the obtained results of this article, the analogous results for the domains of geometric attraction of exponential and Linnik distributions shall be established. More generally, the results may be extended to the domain of geometric attraction of geometrically strictly stable distributions. The extension or generalization of received results will be considered in near future.

\section{COMPETING INTERESTS}

The authors declare that they have no competing interests.

\section{AUTHORS' CONTRIBUTIONS}

All authors contributed equally and significantly to this work. All authors drafted the manuscript, read and approved the final version of the manuscript.

\section{ACKNOWLEDGMENTS}

The authors are greatly indebted to Professor Kozubowski, Tomaz J. from University of Nevada (USA) for providing some his publications related to Geometric Infinitely Divisible (GID) and Geometric Stable (GS) laws. 


\section{REFERENCES}

1. Klebanov LB, Maniya GM, Melamed IA. A problem of Zolotarev and analogs of infinitely divisible and stable distributions in the scheme for summing a random number of random variables. Theory of Probability and Its Applications. 1984;29(4):791-794.

2. Kruglov VM, Korolev VY. Limit theorems for random sums. Moscow: Moskov. Gos. Univ.; 1990.

3. Kalashnikov V. Geometric Sum: Bounds for Rare Events with Applications. Kluwer Academic Publishers; 1997. .

4. Kotz S, Kozubowski TJ, Podrsky K. Springer Science + Business Media, LLC; 2001.

5. Kozubowski TJ, Podrsky K. Asymmetric Laplace Distributions. Mathematical Scientist. 2000

6. Kozubowski TJ. Geometric infinite divisibility, stability, and self-similarity: an overview. Institute of Mathematics, Publish
Academy of Sciences, Warsaw. 2010;p. 39-65.

7. Klebanov LB. Heavy Tailed Distributions. In: Research Gate; 2003.

8. Hung TL. On the rate of convergence in limit theorems for geometric sums. Southeast-Asian J of Sciences. 2013;2(2):117130.

9. Teke SP, Deshmukh SR. On Geometric Infinitely Divisibility. Bulletin of the Marathwada Mathematical Society. December, 2014;15(2):58-64.

10. Sandhya E, Pillai RN. On Geometric Infinitely Divisibility. Journal of the Kerala Statistical Association. December, 1999;10:01-07.

11. Brieman L. Probability. Philadelphia: SIAM; 1992.

12. Petrov VV. Limit Theorems of Probability Theory (Sequences of Independent Random Variables). Clarendon Press Oxford; 1995. . 